\title{
MODERNA PLANTA PILOTO DE AGLOMERAÇÃO A FRIO DE FINOS DE MINÉRIOS DE FERRO EMPREGANDO DISCO PELOTIZADOR SCARABAEUS-1000*
}

\author{
Evandro Moraes da Gama ${ }^{1}$ \\ Abdias Magalhães Gomes ${ }^{2}$ \\ Cláudio Batista Vieira ${ }^{3}$ \\ Guilherme Francisco de Sales Silva ${ }^{4}$ \\ Márcia Batista
}

\section{Resumo}

A aglomeração a frio de minérios, rejeitos de mineração, resíduos sólidos, combustíveis, fertilizantes, produtos químicos, farelos de alimentação animal, etc. é um tema de grande relevância para o setor industrial dentro do contexto de crescimento sustentável. Neste trabalho é feita uma descrição da moderna planta piloto de aglomeração a frio de finos de minérios de ferro empregando o disco pelotizador SC-1000 (Haver). Essa unidade piloto pertence ao Centro de Sustentabilidade da UFMG, que está localizado na cidade de Pedro Leopoldo (Minas Gerais, Brasil). Esse tipo de equipamento difere dos discos tradicionais no mercado por agir de forma dinâmica na formação da pelota crua durante o processo de aglomeração a frio. A tecnologia Scarabeus permite o ajuste automático da altura da borda do disco, assim como da inclinação e da velocidade de rotação, mesmo durante a etapa de operação. É também feita uma abordagem sobre a inovadora tecnologia Scarabaeus de discos pelotizadores para plantas industriais de aglomeração de minérios. Ademais, são apresentadas as principais linhas de pesquisa referentes a aglomeração a frio de materiais finos.

Palavras-chave: Planta piloto; Pelotamento; Disco pelotizador Scarabaeus; Metakaflex.

\section{MODERN COLD AGGLOMERATION PILOT-PLANT OF IRON ORE FINES USING SCARABAEUS-1000 PELLETIZING DISC}

\section{Abstract}

The cold agglomeration of ores, mining tailings, solid wastes, fuel, fertilizers, chemicals components, animal food brans, etc is a topic of great relevance for the industrial sector, as well as for sustainable growth. In this paper, a modern cold agglomeration pilot-plant of iron ore fines, as well as different types of fine materials, using Scarebeus disc (SC-1000 / Haver) is described. This pilot unit is owned by the Sustainability Center of the Federal University of Minas Gerais, which is in Pedro Leopoldo, city located in Minas Gerais (Brazil). Scarabaeus pelletizing disc are maintenance friendly due to their rugged design, allowing a very good process flexibility, high productivity and low energy consumption. This kind of equipment is different from traditional discs because they have a dynamic green pellet formation control. The inclination angle of the disc can be adjusted automatically, even during operation. Both the rotation speed of the disc and the height of the side wall are also automatically adjustable. A general approach about Scarabaeus technology pelletizing disc for industrial agglomeration plants is also presented. Besides, the principal research lines concerning cold agglomeration of fine materials are showed.

Keywords: Pilot-plant; Green ball formation; Scarabaeus pelletizing disc; Metakaflex.

1 Eng. de Minas, Geólogo de Engenharia, Hidrogeólogo, PhD, Prof. Associado do Dep. o de Eng. de Minas da Escola de Eng. da Universidade Federal de Minas Gerais, Belo Horizonte, Minas Gerais, Brasil.

2 Eng. Civil, Dr.,Prof. Associado do Departamento de Eng. de Materiais e Construção da Escola de Engenharia da Universidade Federal de Minas Gerais, Belo Horizonte, Minas Gerais, Brasil.

3 Eng. Metalurgista, M.Sc., Dr., Prof. do Dep. de Eng. Metalúrgica e de Materiais da Escola de Minas da Universidade Federal de Ouro Preto; Prof. da REDEMAT, Ouro Preto, Minas Gerais, Brasil.

4 Estudante do Curso de Eng. Metalúrgica da Universidade Federal de Ouro Preto, Minas Gerais, Brasil.

5 Engenheira de Minas, Especialista associada ao LGG, UFMG, Minas Gerais, Brasil.

* Contribuição técnica ao 44ํ Seminário de Redução de Minério de Ferro e Matérias-primas, 15 Simpósio Brasileiro de Minério de Ferro e 2ํ Simpósio Brasileiro de Aglomeração de Minério de Ferro, 15 a 18 de setembro de 2014, Belo Horizonte, MG, Brasil. 


\section{INTRODUÇÃO}

O pellet feed será o principal e único produto das minerações de ferro do Quadrilátero Ferrífero (MG) a partir de meados da próxima década. Assim, o processo de pelotização terá um papel muito importante no contexto industrial e econômico do estado de Minas Gerais e do Brasil.

Uma das etapas mais importantes da cadeia produtiva de pelotas de minérios de ferro é aquela referente a formação de pelotas cruas, denominada de pelotamento. Apesar dos últimos desenvolvimentos tecnológicos, o pelotamento em escala industrial é um processo que ainda tem forte dependência da sensibilidade do operador.

O conhecimento da influência dos parâmetros granulométricos, químicos, mineralógicos, microestruturais, etc dos minérios, assim como dos tipos de aglomerantes, fundentes, combustíveis, etc na etapa de pelotamento é de vital importância para o processo de pelotização de minérios de ferro. A robustez do equipamento pelotizador e o tipo de controle de processo de pelotamento têm forte influência na qualidade física da pelota crua, assim como nos índices de produtividade e de consumo de energia.

De modo a implementar um maior grau de cientificismo ao processo de pelotamento, a tecnologia Scarabaeus de discos pelotizadores (Haver) foi desenvolvida.Trata-se de uma recente inovação em fabricação de equipamentos de aglomeração a frio, que permite a implementação de um controle dinâmico mais eficiente do fenômeno de conformação de pelotas cruas. A Universidade de Freiberg é atualmente um centro de referência em pesquisas nessa área. No Brasil, a UFMG em conjunto com a UFOP, iniciaram recentemente um amplo programa científico de aglomeração de materiais finos, em parceria com a Haver e outras instituições do Brasil e do exterior. Neste trabalho, descreve-se a planta piloto automatizada de aglomeração a frio de materiais finos, situada no Centro de Sustentabilidade da UFMG em Pedro Leopoldo, empregando o disco pelotizador SC-1000 (Haver). É também feita uma abordagem sobre a tecnologia Scarabaeus de discos pelotizadores para plantas industriais de aglomeração de minérios. Ademais, são apresentadas as principais linhas de pesquisa referentes a aglomeração a frio de finos de materiais.

\section{EQUIPAMENTOS PELOTIZADORES}

\subsection{Introdução}

A conformação de pelotas cruas consiste no rolamento de uma mistura de partículas de pellet feed de minérios de ferro e de finos de fundentes, devidamente umedecida e com adição controlada de aglomerante inorgânico ou orgânico, em um equipamento girante. Os principais equipamentos utilizados na indústria de pelotização de minério de ferro para formação das pelotas cruas são o tambor e o disco pelotizador. No Brasil, a maioria das usinas de pelotização utiliza o disco pelotizador.

O objetivo da etapa de pelotamento é nuclear e produzir pelotas cruas, que são aglomerados esféricos, com uma distribuição de tamanhos e propriedades mecânicas adequadas para os processos subsequentes. 


\subsection{Tambor Pelotizador}

Este equipamento foi desenvolvido a partir do tambor de mistura utilizado em instalações de sinterização e adaptado à sua nova função. Durante os primeiros anos de desenvolvimento, o tambor foi a unidade de pelotização única. Todas as plantas de pelotização operadas em escala industrial nos EUA e na Suécia foram exclusivamente equipadas com tambores até o ano de 1956 [1].

O tambor é composto por um cilindro de movimento rotativo ligeiramente inclinado, aberto em ambas as extremidades, ocasionalmente, com uma pequena retenção do anel ou do cone na extremidade de alimentação, a fim de impedir qualquer fluxo inverso de pelotas na alimentação. No interior do tambor, existe um rolo raspador, de posição fixa, em paralelo ao tambor, cuja função é limitar a altura da camada interna de material.

Para a melhoria da resistência adesiva e da rugosidade, a parede metálica interna lisa é revestida com uma camada de finos úmidos e a sua espessura é controlada e limitada por raspadores. Esses raspadores são estacionários ou de rotação e dispostos em paralelo ao eixo do tambor. Nas instalações modernas, os chamados raspadores espirais rotativos são utilizados, o que não só limita a altura da camada, mas também transmite uma maior resistência imaginária à face interior.

A operação do tambor é considerada simples. A alimentação da mistura a pelotizar devidamente preparada e umedecida é carregada pela extremidade superior do tambor. Se necessário, a água é pulverizada em determinadas áreas para possibilitar melhor formação das pelotas cruas. As partículas da mistura rolam em espirais em direção a extremidade de descarga (Figura 1).

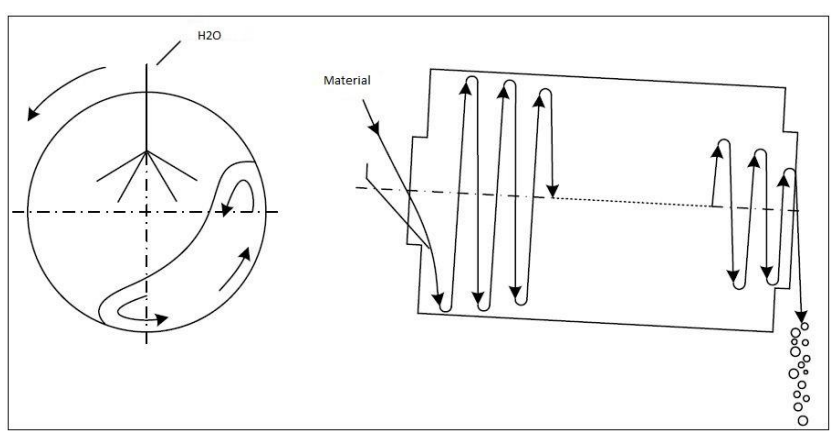

Figura 1: Desenho esquemático mostrando rolamento em espirais das partículas no interior do tambor.

De acordo com o comprimento, a inclinação, a velocidade de rotação e o grau de enchimento do tambor, pelotas cruas com certa distribuição de tamanho são produzidas em um ritmo de produção que é função dos parâmetros de projeto do equipamento, das variáveis de processo e das características intrínsecas do minério. Devido ao modo de operação do tambor, ele não tem praticamente nenhum efeito de classificação. As pelotas descarregadas são classificadas e aquelas com dimensões desejadas são separadas e transportadas para a etapa de processamento térmico. As parcelas de pelotas, acima e abaixo dos limites de especificação granulométrica, retornam para o fluxo de produção para serem novamente processadas com a alimentação do tambor. Assim, sempre existirá material residente no sistema de recirculação e a quantidade desse material tem proporção percentual que varia de $100 \%$ a $400 \%$ da quantidade de material novo que alimenta o tambor, dependendo das condições de operação [1].

As pelotas pequenas contidas no produto de tamanho abaixo do desejado servem, durante a sua passagem repetida através do tambor, como sementes para a formação de pelotas de tamanho adequado. De acordo com a carga circulante

* Contribuição técnica ao 44 Seminário de Redução de Minério de Ferro e Matérias-primas, 15은 Simpósio Brasileiro de Minério de Ferro e $2^{\circ}$ Simpósio Brasileiro de Aglomeração de Minério de Ferro, 15 a 18 de setembro de 2014, Belo Horizonte, MG, Brasil. 
envolvida, a matéria-prima passa repetidamente através do tambor antes das pelotas cruas serem descarregadas. A separação das frações utilizáveis é normalmente conseguida através de um sistema de classificação suave especialmente desenvolvido, de forma a preservar a qualidade da pelota crua e para evitar que a água dos capilares seja pressionada para a superfície.

As flutuações da carga circulante influenciam o grau de enchimento e eficiência do tambor e, em certa medida, o intervalo de tamanho das pelotas. Esta é uma desvantagem da operação do tambor e é ainda objeto de esforços especiais para reduzir ou até mesmo para evitar que isto aconteça. No entanto, isso é um elemento de importante influência, que deve ser considerado na avaliação de todos os outros fatores [1].

\subsection{Disco Pelotizador}

O disco pelotizador foi inicialmente empregado para aglomeração de alimentos e na indústria de fertilizantes antes de sua aplicação na pelotização de minério de ferro, cujas operações se iniciaram aproximadamente em 1950 [1].

O movimento de rolamento é iniciado pela inclinação do fundo liso do disco em vez de ser na parede côncava como no tambor (Figura 2). Devido ao design e a operação do disco, uma classificação granulométrica com relação ao diâmetro dos aglomerados já se inicia durante a formação da pelota. Atualmente, mesmo sendo uma tecnologia mais recente do que a do tambor, uma parte considerável das usinas de pelotização do mundo, utiliza o disco pelotizador como equipamento de pelotamento.
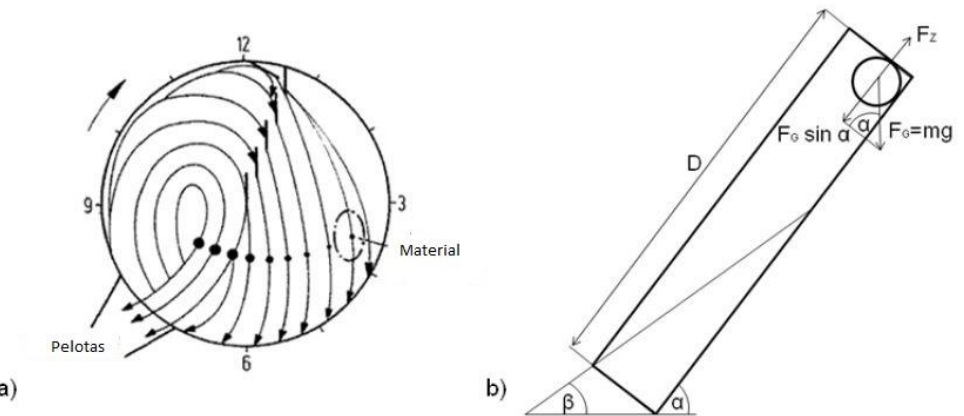

Figura 2: (a) Percurso do material em um disco; (b) Forças atuantes na pelota durante a sua formação.

De um modo semelhante ao do tambor, o disco é constituído por componentes específicos para a conformação da pelota crua. Com uma rotação concêntrica, o disco inclinado é composto por uma área circular de fundo liso. Para assegurar uma resistência ao atrito suficiente e um bom efeito de elevação para a carga de pelotas cruas, uma camada de minério úmido de cerca de poucos centímetros de espessura é empregada na parte inferior do disco (camada de fundo) e é controlada por raspadores.

Diferentes projetos foram desenvolvidos, tais como raspadores fixos e oscilantes ou espirais rotativas. Os discos possuem um dispositivo para controle da sua rotação e inclinação, assim como da taxa de alimentação da mistura a pelotizar. Através do ajuste dos raspadores é possível o controle da espessura de minério formada no fundo disco, de maneira a garantir boas condições para rolamento e aglomeração das partículas da mistura. A capacidade do disco é determinada pela parede lateral posicionada num ângulo de $90^{\circ} \mathrm{em}$ relação à parte inferior. A altura da borda é, em certa medida, dependente do diâmetro do disco. A velocidade angular do disco pode ser ajustada dentro de certos limites, dependendo das propriedades de aglomeração

* Contribuição técnica ao 44 Seminário de Redução de Minério de Ferro e Matérias-primas, 15ํ Simpósio Brasileiro de Minério de Ferro e $2^{\circ}$ Simpósio Brasileiro de Aglomeração de Minério de Ferro, 15 a 18 de setembro de 2014, Belo Horizonte, MG, Brasil. 
das matérias-primas empregadas $\mathrm{O}$ ponto de alimentação da mistura de materiais a pelotizar no disco também exerce grande influência no fenômeno de formação das pelotas cruas e pode ser ajustado em função dos resultados operacionais [1].

A disposição e a forma de operação do disco pelotizador permitem a classificação granulométrica das pelotas cruas, devido a combinação da força centrífuga e do próprio peso dos aglomerados produzidos. A mistura de materiais a pelotizar é alimentada numa posição específica do equipamento e as pelotas em formação sofrem rolamentos sucessivos sobre as partículas finas alimentadas, contribuindo para crescimento gradativo do tamanho desses aglomerados. Os raspadores também funcionam como direcionadores de fluxo, definindo a trajetória das pelotas de diferentes tamanhos até sua descarga do disco, na região oposta a aquela de alimentação da mistura. Posteriormente, os aglomerados produzidos são classificados na faixa granulométrica estabelecida pelo processo. As pelotas cruas que estão fora da faixa de especificação retornam ao sistema de pelotamento e são reprocessadas no disco juntamente com a mistura de materiais a pelotizar.

A principal variável de processo a ser controlada na etapa de pelotamento é tempo de residência da mistura de materiais a pelotizar no interior do disco. Esse parâmetro é influenciado por diversos fatores, tais como, velocidade de rotação do disco, inclinação do equipamento, taxa de alimentação da mistura de materiais, ponto de alimentação da carga no disco, espessura da camada de fundo e características de projeto do disco.

\subsection{Aglomerantes}

O uso de aglomerantes minerais e orgânicos na etapa de formação da pelotas cruas é uma prática consolidada pelas usinas de pelotização de minério de ferro [2].

As principais funções da adição de agentes aglomerantes na mistura a pelotizar são:

- Aumentar a viscosidade da fase líquida dentro dos capilares;

- Manter a coesão das pelotas cruas, e assim garantir a plasticidade do minério úmido, possibilitando a nucleação de sementes até a formação das pelotas cruas;

- Aumentar a resistência das pelotas cruas secas em comparação com as pelotas úmidas e manter sua estabilidade física até que ocorra a reação de sinterização das partículas na etapa de queima.

O aglomerante mineral mais empregado em pelotização de minério de ferro é a bentonita e sua aplicação é maior na fabricação de pelotas para altos-fornos. Tratase de um material tixotrópico, ou seja, suas placas separam-se quando em contato com a água, formando um gel coloidal. Esse gel diminui a distância entre as partículas de pellet feed aumentando a intensidade das forças de Van der Waals e, em consequência, confere resistência mecânica as pelotas cruas.

A bentonita é um argilo mineral, tendo como principal constituinte mineralógico a montmorilonita. É um material com alto teor de sílica e sua dosagem (\% em peso) na pelota crua é da ordem de $0,5 \%$ a $0,7 \%$. Seu uso implica na incorporação de sílica e de alumina na pelota queimada.

A cal também tem sido usada como agente aglomerante em processos de pelotização de minérios de ferro.

Os aglomerantes orgânicos são compostos poliméricos (longas cadeias de carbono de alta massa molecular). Apresentam custos mais altos e são utilizados preferencialmente na fabricação de pelotas de minérios de ferro para os processos de redução direta. Seu uso tem como principal vantagem não incorporar

\footnotetext{
* Contribuição técnica ao $44^{\circ}$ Seminário de Redução de Minério de Ferro e Matérias-primas, 15은 Simpósio Brasileiro de Minério de Ferro e $2^{\circ}$ Simpósio Brasileiro de Aglomeração de Minério de Ferro, 15 a 18 de setembro de 2014, Belo Horizonte, MG, Brasil.
} 
contaminantes às pelotas, pois são volatilizados durante o processamento térmico.

Os principais tipos comerciais de aglomerantes orgânicos são o Peridur (CMC carboximetilcelulose), Polybinder (CMC - carboximetilcelulose), Carbinder (HEC hidroxietilcelulose) e Alcotac (PAM - poliacrilamida). As dosagens típicas utilizadas estão compreendidas na faixa de 350 a 600 gramas/tonelada de mistura.

Mais recentemente, tem se iniciado no Brasil estudos em escala piloto para avaliar o uso do ligante Metakaflex em substituição parcial ou total da bentonita em processos de pelotização de minérios hematíticos. O Metakaflex é um ecoproduto fabricado a partir da calcinação flash de estéreis argilosos [4,5].

\section{DESENVOLVIMENTO DA TECNOLOGIA SCARABAEUS}

A Tecnologia Scarabaeus (Ver Figura 3) foi desenvolvida pelo Grupo Haver \& Boecker, que possui 20 anos de experiência como fornecedores de discos de alta capacidade operando na produção de pelotas cruas e de microaglomerados de diversas plantas industriais de pelotização e de sinterização, tais como os processos traveling-grate, grate-kiln e HPS (hybrid pelletized sínter).
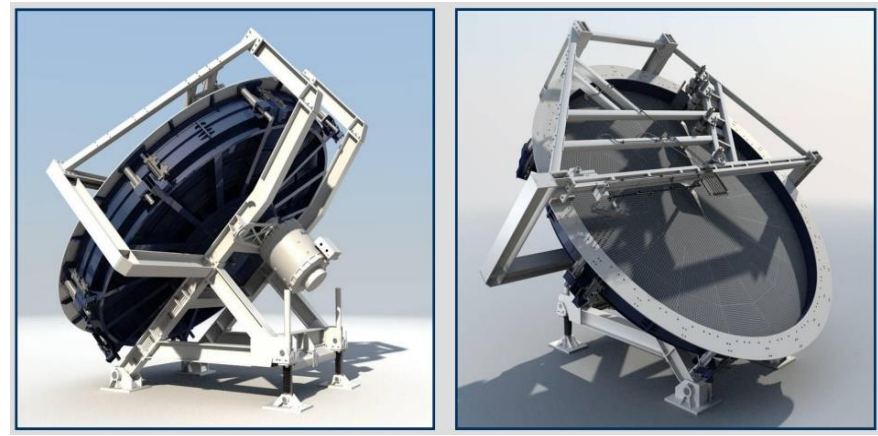

Figura 3: Imagem frontal e traseira do disco pelotizador SC7500

Desde 2008 o Grupo Haver \& Boecker tem promovido o desenvolvimento de novas tecnologias na área de aglomeração de finos no centro de pesquisa e desenvolvimento-Haver Engineering Meißen (HEM) em parceria com a Universidade TU Bergakademie Freiberg na Alemanha. O disco Scarabaeus é o resultado de uma série de avançados estudos de investigação em equipamento de pelotização para descobrir as necessidades da arte de pelotizar [6].

A inovação confere ao equipamento um sistema operacional flexível de fácil manutenção, com a adição de uma variável tridimencional, controle de inclinação automático e novo acionamento. O controle dinâmico das variáveis do equipamento age de modo a minimizar o impacto sobre a produção e qualidade do produto quanto as flutuações e mudanças no processo (ver Figura 4).

Toda essa flexibilidade faz com que o disco pelotizador Scarabeus seja uma solução tecnológica para o desenvolvimento de novas aplicações e desafios de otimização de processo na área de aglomeração de materiais finos.

No centro de pesquisa HEM, localizado na TU Bergakademie Freiberg, são realizados ensaios e estudos na planta de pelotização piloto com capacidade de até 1,0 t/h para determinar a influência dos paramêtros de ajuste do disco, desenvolver novos ligantes (orgânicos e minerais), otimizar misturas e etc. Diferentes tipos de materiais podem ser ensaiados (Ver Figura 5).

A ideia é desenvolver um sistema de controle auto-ajustável das principais variáveis que envolvem o processo de pelotização, tais como, inclinação do disco, velocidade de rotação, umidade da pelota crua e altura da borda lateral (Ver Figuras 6 e 7).

* Contribuição técnica ao 44ํ Seminário de Redução de Minério de Ferro e Matérias-primas, 15은 Simpósio Brasileiro de Minério de Ferro e 2 Simpósio Brasileiro de Aglomeração de Minério de Ferro, 15 a 18 de setembro de 2014, Belo Horizonte, MG, Brasil. 


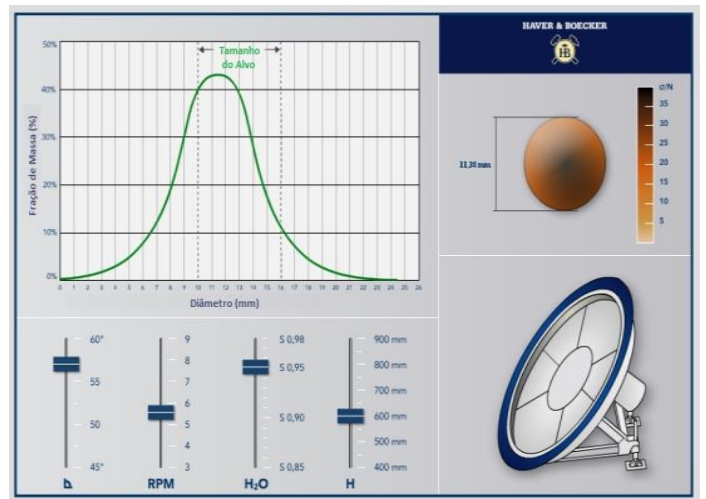

Figura 4: Interface de ajuste automático dos parâmetros do disco pelotizador SC7500

Diversos modelos de discos pelotizadores empregando a tecnologia Sacarabeus estão disponíveis no mercado, conforme descrição detalhada apresentada na Tabela 1.

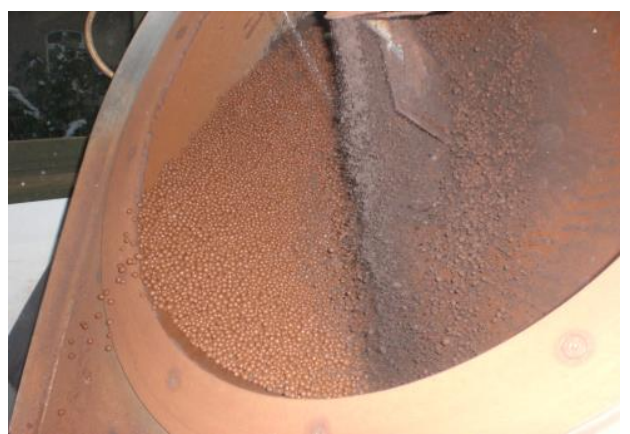

(a) Ensaios com minério de ferro

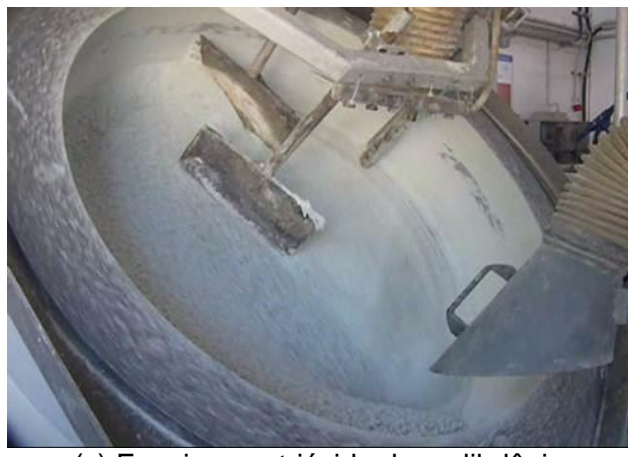

(c) Ensaio com trióxido de molibdênio

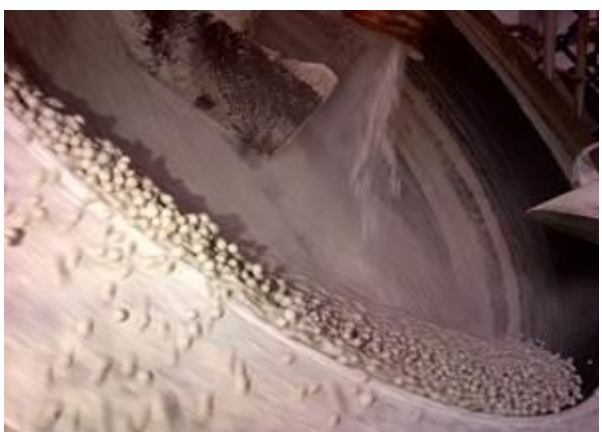

(b) Ensaios com matéria-prima para indústria de vidro

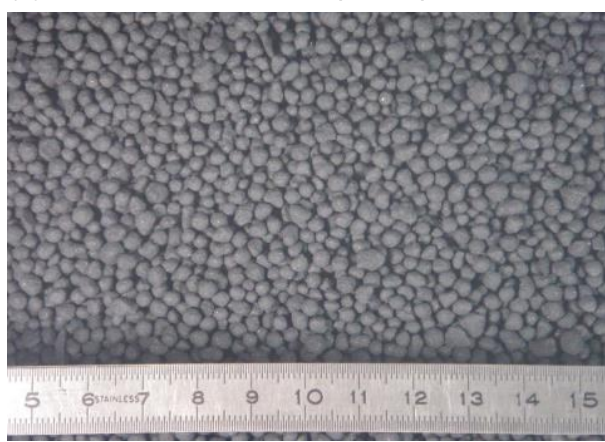

(d) Produção de pelotas cruas de minério de ferro

Figura 5: Ensaios de pelotamento usando disco SC1000 com diferentes tipos de materiais

Tabela 1 - Série de discos pelotizadores com tecnologia Scarabeus: modelos disponíveis no mercado

\begin{tabular}{|c|c|c|c|c|c|c|c|c|}
\hline Machine type & & SC500 & SC1000 & SC2200 & SC3200 & SC4200 & SC6000 & SC7500 \\
\hline Diâmetro do Disco & $\mathrm{mm}$ & 500 & 1000 & 2200 & 3200 & 4200 & 6000 & 7500 \\
\hline $\begin{array}{l}\text { Altura da Borda Lateral } \\
\text { (Fixo) }\end{array}$ & $\mathrm{mm}$ & $\begin{array}{c}\text { Não } \\
\text { Disponível }\end{array}$ & $\begin{array}{c}\text { Não } \\
\text { disponível }\end{array}$ & $\max .500$ & $\max .700$ & $\max .800$ & $\max .900$ & $\max .900$ \\
\hline $\begin{array}{c}\text { Altura da Borda Lateral } \\
\text { (Ajustável) }\end{array}$ & $\mathrm{mm}$ & - & $240-400$ & $250-450$ & $300-550$ & $400-750$ & $500-900$ & $500-900$ \\
\hline Rotação Nominal & rpm & 80 & 35 & 20 & 20 & 10 & 7 & 6 \\
\hline Área & $\mathrm{m}^{2}$ & 0,2 & 0,8 & 3,8 & 8,0 & 13,8 & 28,3 & 44,1 \\
\hline Peso & ton & - & 1,5 & $5 \mathrm{t}$ & 12 & 16,5 & 45 & 55 \\
\hline
\end{tabular}

* Contribuição técnica ao 44 Seminário de Redução de Minério de Ferro e Matérias-primas, $15^{\circ}$ Simpósio Brasileiro de Minério de Ferro e $2^{\circ}$ Simpósio Brasileiro de Aglomeração de Minério de Ferro, 15 a 18 de setembro de 2014, Belo Horizonte, MG, Brasil. 

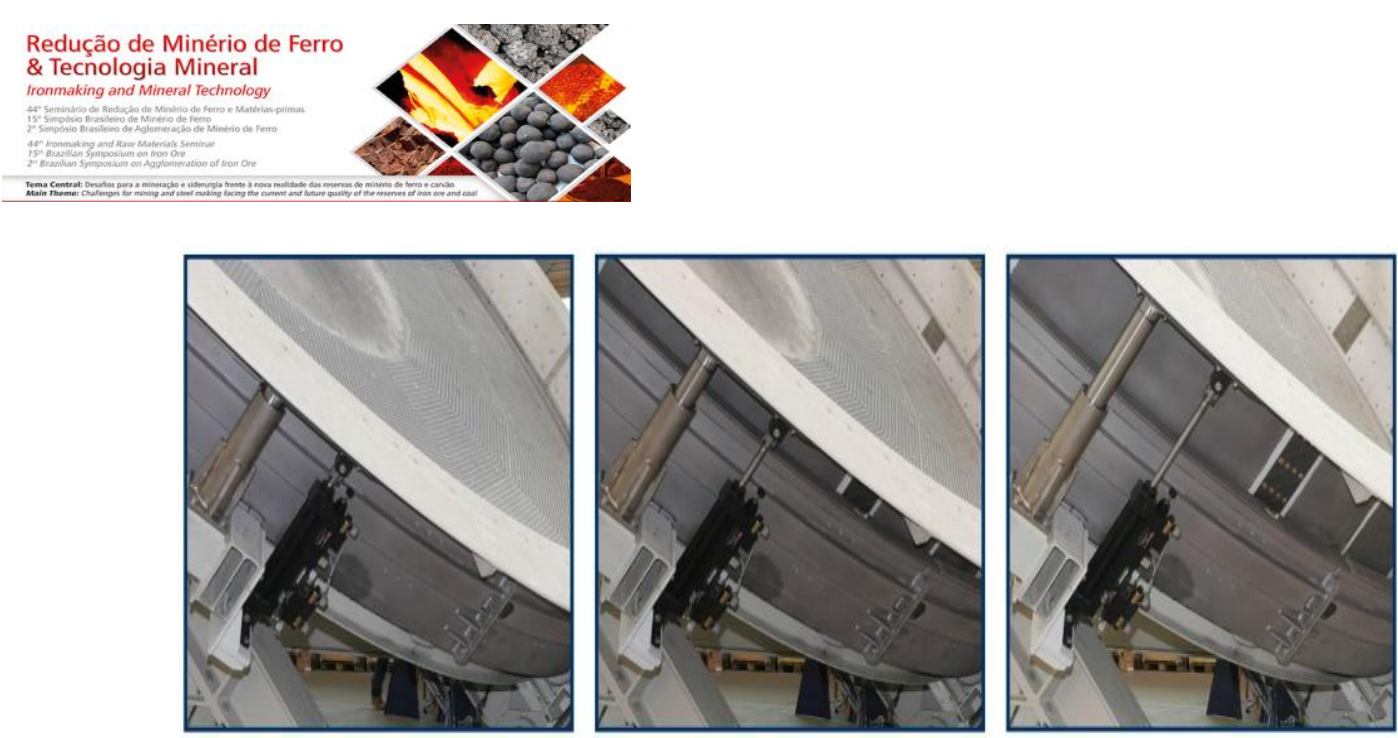

Figura 6: Detalhe do ajuste tridimencional do disco pelotizador SC7500. Flexibilidade no tempo de retenção das pelotas durante o fluxo contínuo do material de alimentação.

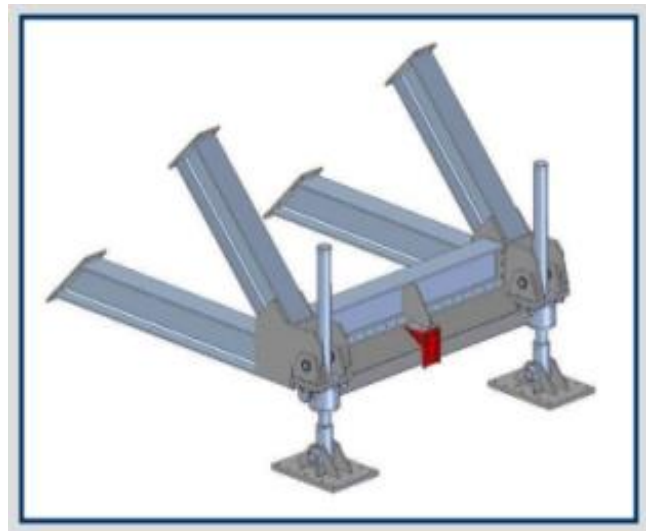

Figura 7: llustração do ajuste da inclinação do disco SC7500.

\section{PLANTA-PILOTO DE AGLOMERAÇÃO A FRIO: SC1000}

O Laboratório de Geotecnologia \& Geomateriais do Centro de Sustentabilidade (LGG) da UFMG, localizado em Pedro Leopoldo/MG, conta atualmente com uma planta piloto integrada de calcinação flash e de pelotamento de materiais finos. $O$ LGG mantém parceria a nível acadêmico e científico com diversas instituições brasileiras e internacionais, tais como, UFOP (Departamento de Engenharia Metalúrgica e de Materiais), INSA - Institut des Sciences Appliques de Toulouse, TU Bergakademie Freiberg, Haver \& Boecker Latino Americana, etc.

Diversos tipos de materiais podem ser processados na planta piloto flash, tais como, estéreis argilosos, rejeitos de mineração, calcário, minérios de alto PPC (perda por calcinação), etc.

Na planta piloto de pelotamento é possível efetuar estudos de aglomeração a frio de finos de diferentes tipos de materiais, tais como, minério de ferro, minério de manganês, carvão vegetal, resíduos industriais, rejeitos de mineração, produtos de alimentação animal, fertilizantes, etc.

A unidade piloto de pelotamento conta com a seguinte infraestutura:

- Um pelotizador de bancada para estudos preliminares (no LGG);

- Um disco pelotizador SC1000 (no LGG);

- Dois misturadores (no LGG);

- Dosador eletromecânico para alimentação da mistura a pelotizar (no LGG);

- Laboratório de caracterização física das pelotas cruas (no LGG);

- Laboratórios para caracterização química, mineralógica e termodinâmica de materiais (na EEUFMG e na EMUFOP). 
equipamento SC1000, mostrado nas Figuras 8 e 9, é um modelo de disco com diâmetro de 1,0 metro e com capacidade de produção de até 1,0 tonelada/hora de pelotas cruas. Ele permite o ajuste automático da altura da borda durante a sua operação, permitindo variar de $240 \mathrm{~mm}$ a $400 \mathrm{~mm}$. Existe a possibilidade de regulagem automática da inclinação durante a operação, podendo variar de $35^{\circ}$ a $65^{\circ}$. É também possível o ajuste automático da velocidade de rotação, podendo variar de 2 rpm a 48 rpm.

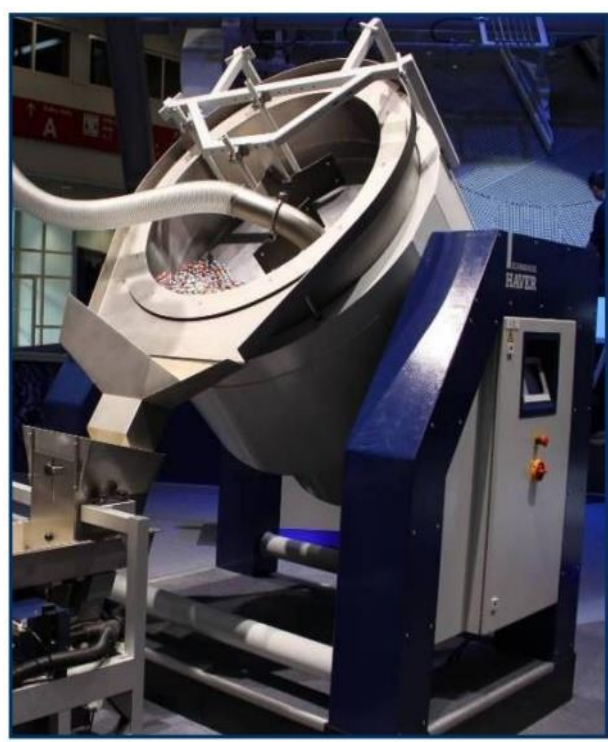

Figura 8: Vista do disco pelotizador SC1000.

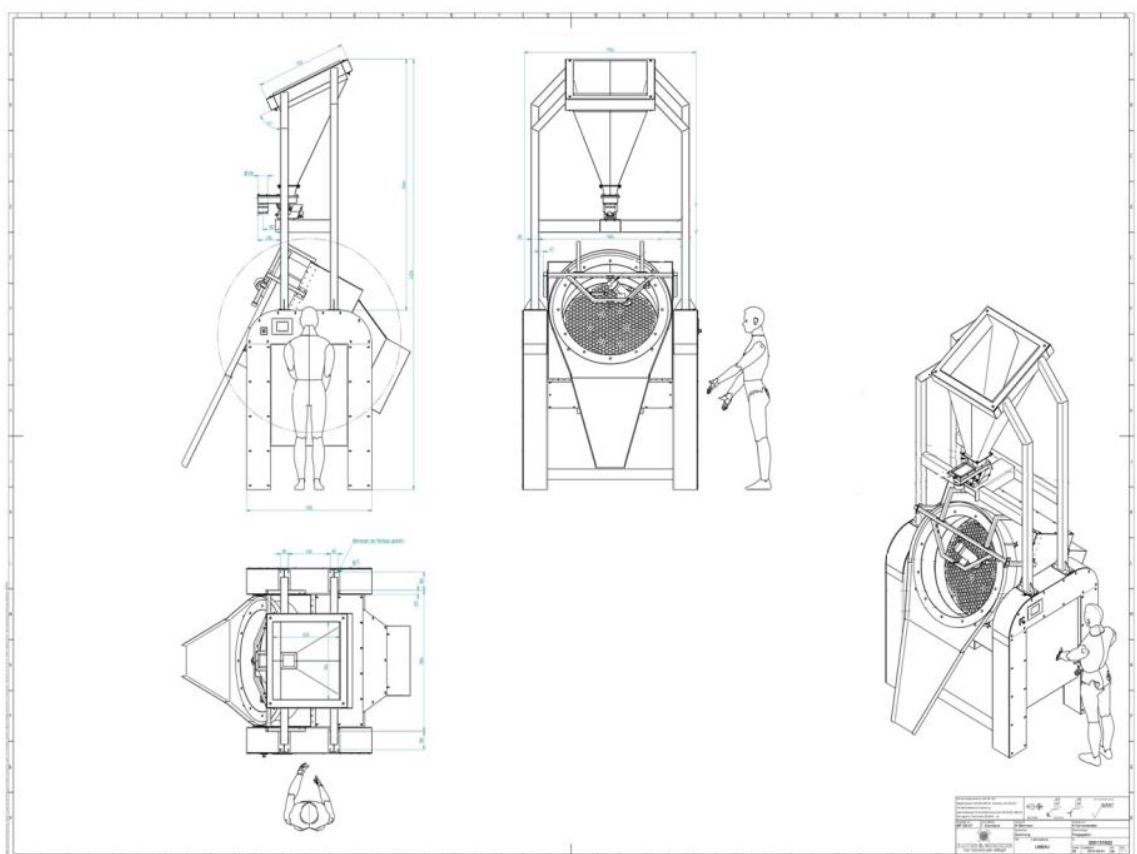

Figura 9: Detalhes do sistema alimentador e do disco SC1000 na planta piloto do LGG/UFMG.

$\mathrm{Na}$ Tabela 2 é apresentada uma lista das principais linhas de pesquisa do LGG com relação a aglomeração a frio de materiais finos, envolvendo pesquisadores brasileiros da UFMG e UFOP. Uma das principais linhas se refere ao uso no pelotamento de minérios do ligante Metakaflex, produzido a partir da calcinação flash de estéreis argilosos [4,5].

* Contribuição técnica ao 44 Seminário de Redução de Minério de Ferro e Matérias-primas, 15은 Simpósio Brasileiro de Minério de Ferro e $2^{\circ}$ Simpósio Brasileiro de Aglomeração de Minério de Ferro, 15 a 18 de setembro de 2014, Belo Horizonte, MG, Brasil. 
Tabela 2 - Principais linhas de pesquisa do LGG: aglomeração a frio de materiais finos.

\begin{tabular}{|c|c|c|}
\hline $\begin{array}{l}\text { Tecnologia/Material } \\
\text { a ser Estudado }\end{array}$ & $\begin{array}{c}\text { Principais Estudos - Linhas de } \\
\text { Pesquisas }\end{array}$ & Aplicações \\
\hline $\begin{array}{c}\text { Tecnologia } \\
\text { Scarabaeus de } \\
\text { aglomeração a frio }\end{array}$ & $\begin{array}{l}\text { Uso da tecnologia Scarabaeus para } \\
\text { aglomeração a frio de materiais finos }\end{array}$ & $\begin{array}{l}\text { Metalurgia extrativa, indústria de } \\
\text { fertilizantes, termoelétricas, indústria } \\
\text { de alimentos, construção civil, etc. }\end{array}$ \\
\hline Metakaflex & $\begin{array}{l}\text { Estudo de aglomeração a frio de finos } \\
\text { de minérios e de combustíveis sólidos } \\
\text { utilizando Metakaflex como } \\
\text { aglomerante }\end{array}$ & $\begin{array}{l}\text { Processos de pelotização e de } \\
\text { sinterização de minérios (ferrosos e } \\
\text { não ferrosos). } \\
\text { Fornos elétricos para fabricação de } \\
\text { ferro-ligas. }\end{array}$ \\
\hline Carvão Vegetal & $\begin{array}{l}\text { Desenvolvimento de pelotas de cura } \\
\text { a frio de finos de carvão vegetal }\end{array}$ & $\begin{array}{l}\text { Fornos elétricos para fabricação de } \\
\text { ferro-ligas. } \\
\text { Churrasqueiras. } \\
\text { Caldeiras em Centrais Termoelétricas }\end{array}$ \\
\hline Carvão Mineral & $\begin{array}{l}\text { Desenvolvimento de pelotas de cura } \\
\text { a frio de carvões minerais }\end{array}$ & Caldeiras em Centrais Termoelétricas \\
\hline $\begin{array}{l}\text { Minério de } \\
\text { Manganês }\end{array}$ & $\begin{array}{l}\text { Desenvolvimento de pelotas de cura } \\
\text { a frio de finos de minério de } \\
\text { manganês }\end{array}$ & $\begin{array}{l}\text { Fornos elétricos para fabricação de } \\
\text { ferro manganês. }\end{array}$ \\
\hline $\begin{array}{c}\text { Minérios } \\
\text { (Não Ferrosos) }\end{array}$ & $\begin{array}{l}\text { Estudo de pelotamento de finos de } \\
\text { minérios (não ferrosos) }\end{array}$ & $\begin{array}{l}\text { Processos de aglomeração de } \\
\text { minérios (não ferrosos) } \\
\text { Fornos elétricos para fabricação de } \\
\text { ferro-ligas. }\end{array}$ \\
\hline $\begin{array}{l}\text { Minério de Ferro } \\
\text { (finos) }\end{array}$ & $\begin{array}{l}\text { Desenvolvimento de pelotas de cura } \\
\text { a frio de finos de minério de ferro }\end{array}$ & $\begin{array}{l}\text { Fornos elétricos para fabricação de } \\
\text { ferro-ligas. }\end{array}$ \\
\hline $\begin{array}{l}\text { Minério de Ferro } \\
\text { (Pellet Feed) }\end{array}$ & $\begin{array}{l}\text { Estudo da formação de pelotas cruas } \\
\text { de concentrados de minérios de ferro } \\
\text { hematíticos, magnetíticos e } \\
\text { goethíticos. }\end{array}$ & $\begin{array}{l}\text { Processos traveling-grate e grate-kiln } \\
\text { de pelotização de minério de ferro }\end{array}$ \\
\hline $\begin{array}{l}\text { Minério de Ferro } \\
\text { (Mistura de Sinter } \\
\text { Feed e Pellet } \\
\text { Feed) }\end{array}$ & $\begin{array}{l}\text { Estudo da aglomeração a frio em } \\
\text { disco pelotizador de misturas de } \\
\text { sínter feed e pellet feed }\end{array}$ & $\begin{array}{l}\text { Uso em processos de sinterização de } \\
\text { minério de ferro (processo HPS - } \\
\text { Hybrid Pelletizing Sinter) }\end{array}$ \\
\hline $\begin{array}{l}\text { Aglomerantes } \\
\text { Orgânicos e } \\
\text { Minerais }\end{array}$ & $\begin{array}{l}\text { Avaliação do comportamento de } \\
\text { diferentes tipos de aglomerantes } \\
\text { orgânicos e minerais em pelotamento } \\
\text { de minérios de ferro }\end{array}$ & $\begin{array}{l}\text { Processos de pelotização de minério } \\
\text { de ferro. }\end{array}$ \\
\hline $\begin{array}{l}\text { Fertilizantes } \\
\text { Agricolas } \\
\end{array}$ & Pelotamento de fertilizantes & Agricultura \\
\hline $\begin{array}{l}\text { Rejeitos de } \\
\text { Mineração }\end{array}$ & Pelotamento de rejeitos de mineração & Siderurgia \\
\hline $\begin{array}{l}\text { Resíduos Sólidos } \\
\text { Siderúrgicos }\end{array}$ & $\begin{array}{l}\text { Pelotamento de resíduos sólidos } \\
\text { siderúrgicos }\end{array}$ & Siderurgia \\
\hline $\begin{array}{l}\text { Farelo de } \\
\text { Alimentação } \\
\text { Animal }\end{array}$ & $\begin{array}{l}\text { Pelotamento de farelos de } \\
\text { alimentação animal }\end{array}$ & Mercado de nutrição animal. \\
\hline
\end{tabular}

\section{CONSIDERAÇÕES FINAIS}

O LGG - Laboratório de Geotecnologia \& Geomateriais do Centro de Sustentabilidade da UFMG está capacitado para realização de estudos avançados em unidade piloto de aglomeração a frio, utilizando a tecnologia Scarabaeus, para processamento de finos de materiais minerais (ferrosos e não ferrosos), combustíveis sólidos, rejeitos de mineração, resíduos industriais, farelos de alimentação animal, etc. 
O controle dinâmico de formação de pelotas cruas empregando a tecnologia Scarabaeus propicia excelentes ganhos de produtividade, redução do consumo energético e melhoria da qualidade intrínseca do produto obtido, além de permitir introduzir maior grau de cientificismo ao processo. É possível desenvolver soluções tecnológicas customizadas para cada tipo de material a ser estudado.

O LGG integra diferentes tecnologias para aproveitamento de passivos ambientais de mineração para produção de ligantes de baixo custo. O Metakaflex é o principal ecoproduto obtido a partir da calcinação flash de estéreis argilosos e com aplicações diversas (argamassa, pavimentação, construção civil, etc), além do grande potencial de uso em processos de pelotização de minérios.

\section{Agradecimentos}

A FAPEMIG - Fundação de Amparo a Pesquisa de Minas Gerais pelo financiamento do projeto de pesquisa referente ao desenvolvimento do ligante Metakaflex (Projeto Tec rdp 00088-10 160305, 2011-2015).

A Haver \& Boecker Latino Americana pelo apoio ao LGG-UFMG.

\section{REFERÊNCIAS}

1 Meyer K. Pelletizing of Iron Ores. Berlin: Springer-Verlag, 1980.

2 Eisele TC, Kawatra SK. A Review of Binders in Iron Ore Pelletization. Mineral Processing \& Extractive Metall. Rev. Taylor \& Francis, Michigan, v.24, p.1-90, 2003.

3 Gama EM. inventor; Universidade Federal de Ouro Preto, Cessionário. Processo de preparação de metakflex aglomerante de alta resistência de produtos e processos que venham a utilizar metakflex. Patente no Brasil, PI 0604142-6A. 22 de Abril de 2008.

4 Gama EM, Gomes AM, Matias GP, Vieira CB. Planta piloto de produção de Metakaflex e seu uso como aglomerante no pelotamento de minério de ferro. A ser publicado no Anais do $2^{\circ}$ Simpósio Brasileiro de Aglomeração de Minério de Ferro da ABM. Belo Horizonte, Setembro de 2014.

5 Silva GS. Calcinação flash de lamas de barragem de rejeito para a produção de metakflex e o seu emprego como aglomerante no pelotamento de concentrados de minérios itabiríticos. Proposta de monografia de final de curso de engenharia metalúrgica. Universidade Federal de Ouro Preto. 59p. 2014.

6 www.haverengineering.de; Haver Engineering $\mathrm{GmbH}$, An-Institut der TU Bergakademie Freiberg. Meißen/Deutschland - Germany. 2014. 